\title{
Metabolic Risk Factors of Alzheimer's Disease, Dementia with Lewy Bodies, and Normal Elderly: A Population-Based Study
}

\author{
Chih-Kuang Cheng $\mathbb{D},{ }^{1}$ Yu-Chien Tsao $\mathbb{D},{ }^{2,3,4}$ Yuan-Chih Su $\mathbb{D},{ }^{5,6}$ Fung-Chang Sung $\mathbb{D},{ }^{7}$ \\ Hsu-Chih Tai $\mathbb{D}^{8}{ }^{8}$ and Woon-Man Kung $\mathbb{D}^{8,9,10}$ \\ ${ }^{1}$ Stroke Center and Department of Neurology, Linkou Medical Center, Chang Gung Memorial Hospital and College of Medicine, \\ Chang Gung University, Taoyuan, Taiwan \\ ${ }^{2}$ Department of Internal Medicine, Yonghe Cardinal Tien Hospital, Taipei, Taiwan \\ ${ }^{3}$ Department of Neurology, Neurological Institute, Taipei Veterans General Hospital, Taipei, Taiwan \\ ${ }^{4}$ School of Medicine, National Yang-Ming University, Taipei, Taiwan \\ ${ }^{5}$ Management Office for Health Data, China Medical University Hospital, Taichung, Taiwan \\ ${ }^{6}$ College of Medicine, China Medical University, Taichung, Taiwan \\ ${ }^{7}$ Department of Public Health, China Medical University, Taichung, Taiwan \\ ${ }^{8}$ Department of Exercise and Health Promotion, College of Education, Chinese Culture University, Taipei, Taiwan \\ ${ }^{9}$ Division of Neurosurgery, Department of Surgery, Taipei Tzu Chi Hospital, Buddhist Tzu Chi Medical Foundation, \\ New Taipei City, Taiwan \\ ${ }^{10}$ Department of Surgery, School of Medicine, Buddhist Tzu Chi University, Hualien, Taiwan
}

Correspondence should be addressed to Woon-Man Kung; nskungwm@yahoo.com.tw

Received 10 February 2018; Revised 17 April 2018; Accepted 9 May 2018; Published 3 June 2018

Academic Editor: Jan Aasly

Copyright (C) 2018 Chih-Kuang Cheng et al. This is an open access article distributed under the Creative Commons Attribution License, which permits unrestricted use, distribution, and reproduction in any medium, provided the original work is properly cited.

\begin{abstract}
Background. Alzheimer's disease (AD) and dementia with Lewy bodies (DLB) share many risk factors. Evidence suggests that metabolic risk factors are important to AD; however, their association with DLB is unclear. The risk of cardiovascular diseases (CVD) associated with AD and DLB is also uncertain. Thus, this nationwide, population-based study was designed to evaluate the metabolic and CVD risks in AD and DLB. Materials and Methods. Data were obtained from the Taiwan National Health Insurance Research Database. AD patients, DLB patients, and normal control (NC) individuals from 1996 to 2013 were enrolled for risk assessment. Results. In total, 7544 NC individuals, 1324 AD patients, and 562 DLB patients were enrolled. Participants with one or more metabolic risk factors had significantly higher odds of AD or DLB. No significant differences in metabolic risk factors were observed between DLB and AD patients. AD patients had a lower risk of CVD ( $\mathrm{aHR}=0.67,95 \% \mathrm{CI}=0.59-0.76$, $p$ value $<0.001)$ and coronary artery disease $(\mathrm{CAD})(\mathrm{aHR}=0.59,95 \% \mathrm{CI}=0.51-0.69, p$ value $<0.001)$ than NC. DLB patients had a higher risk of ischemic stroke $(\mathrm{aHR}=2.27,95 \% \mathrm{CI}=1.68-3.06, p$ value $<0.001)$ than NC. Conclusion. Metabolic risk factors are important in $\mathrm{AD}$ and $\mathrm{DLB}$. Patients with $\mathrm{AD}$ might have a lower risk of $\mathrm{CAD}$ and ischemic strokes. Patients with DLB might have a higher risk of ischemic stroke.
\end{abstract}

\section{Introduction}

Alzheimer's disease (AD) and dementia with Lewy bodies (DLB) are leading causes of dementia [1]. AD is caused by progressive neurodegeneration in the medial temporal lobe, hippocampus formation, and other brain areas with synergistic amyloid and tau proteinopathy $[1,2]$. The risk factors for sporadic $\mathrm{AD}$ are complex. Apolipoprotein E $\varepsilon 4$ allele (APOE4) is one of best-known genetic risk factors. Additional risk factors include age, family history of $\mathrm{AD}$, previous stroke, sleep apnea, traumatic brain injury (TBI), and limited education [1]. DLB is characterized by the propagation of aggregated intracellular $\alpha$-synuclein (Lewy bodies) [3]. Risk factors of DLB overlap with that of AD to some extent; for 
example, APOE4, previous stroke, and TBI are risk factors of both DLB and AD [1, 4-6].

Emerging evidence suggests that metabolic risk factors contribute not only to cardiovascular diseases (CVD) like coronary artery disease (CAD), and ischemic strokes, such as cerebrovascular accident (CVA), but also to dementia [7-9]. Hypertension, diabetes mellitus (DM), and hyperlipidemia are known risk factors for $\mathrm{AD}[9,10]$. Moreover, a history of CAD or CVA is associated with accelerated cognitive decline in $\mathrm{AD}$ patients $[8,11,12]$.

However, it is unclear if metabolic risks are also important risk factors of DLB, and it is unclear if AD and DLB patients are more susceptible to CVD. Hence, we conducted a nationwide, population-based, normal control, risk-adjusted, retrospective cohort study in Taiwan to investigate the metabolic risks and the incidence of CVD in patients with $\mathrm{AD}$ and DLB. Our purpose is to determine the modifiable metabolic risk factors in DLB and the risk of CVD in AD and DLB to improve the prevention of dementia and CVD.

\section{Materials and Methods}

2.1. Study Design. We conducted a retrospective cohort study using health insurance data from the National Health Insurance Research Database (NHIRD), which contained medical information of over $99 \%$ of Taiwan citizens. The Longitudinal Health Insurance Database 2000 (LHID2000), a subdataset of the NHIRD, was applied for this study. The LHID2000 contains diagnostic data of the International Classification of Diseases, 9th Revision, Clinical Modification (ICD-9-CM) code, and treatment data from the outpatient visits and hospitalizations from 1996 to 2013 of 1 million random individuals. All participants were encrypted with a dummy number. This study was approved by the Institutional Review Board and the Ethics Committee of China Medical University Hospital, Taiwan (CMUH104-REC2-115 (CR-2)).

This study investigated AD patients, DLB patients, and normal control (NC) individuals who were enrolled. Both $\mathrm{AD}$ and $\mathrm{DLB}$ patients were identified using specific criteria. $\mathrm{AD}$ patients who met any of the following criteria were included: (1) the ICD-9-CM code 331.0 (AD diagnosis date defined as the index date), or (2) having a diagnosis record of dementia (ICD-9-CM: 290.0-290.4, 294.1, and 331.0$331.2)$ and receiving acetylcholinesterase inhibitors or memantine treatment (dementia diagnosis date defined as the index date). The exclusion criteria for the $\mathrm{AD}$ cohort were patients with (1) any records of cognitive impairment (ICD-9-CM: 331.8, 331.83, and 331.9), Huntington's disease (ICD-9-CM: 333.4), Creutzfeldt-Jakob disease (ICD-9-CM: 046.11 and 046.19), and hydrocephalus (ICD-9-CM: 331.3, $331.4,331.5,741.0$, and 742.3), (2) a history of stroke (ICD9-CM: 430-435) or CAD (ICD-9-CM: 410-414) before the index date, and (3) Parkinson's disease (PD) or Parkinsonism diagnosis recorded within 1 year after the index date. For DLB patients, the inclusion criteria were (1) the diagnosis of PD and Parkinsonism (ICD-9-CM: 332, 332.0, 332.1, $333,333.0$, and 333.9) 1 year after diagnosis of dementia (ICD-9-CM: 290.0-290.3, 294.1, and 331.0) (dementia diagnosis date defined as the index date), (2) diagnosis of dementia within 1 year after diagnosis of PD and Parkinsonism (dementia diagnosis date defined as the index date), and (3) ICD-9-CM code 331.82 (DLB diagnosis date defined as the index date). The exclusion criteria for DLB patients included a history of stroke, CAD, head injury (ICD-9-CM: 850-854 and 959.01), or hydrocephalus. Patients with the diagnosis of both $\mathrm{AD}$ and DLB were excluded from the study cohorts. The NC population was defined as patients without the diagnosis of $\mathrm{AD}, \mathrm{DLB}$, other dementias, or Parkinson's disease. The NC population excluded individuals with a history of stroke or CAD. The study population selection procedure is shown in Figure 1.

2.2. Statistical Analysis. The main outcomes of this study were stroke (ICD-9-CM: 430-435) and CAD (ICD-9-CM: 410-414) after the index date. Only inpatient records were used to identify patients with stroke. A history of hypertension (ICD-9-CM: 401-405), diabetes mellitus (ICD-9-CM: 250), and hyperlipidemia (ICD-9-CM: 272) were included as comorbidities. All comorbidities were considered to be significant after at least one inpatient visit or two outpatient visits. In addition, we investigated the use of aspirin, clopidogrel, and warfarin before the index date. Demographic characteristics of sex and age were considered as covariates. We combined the AD and DLB cohorts and matched them with the NC population according to sex and age in a $1: 4$ ratio by propensity score matching. This study compared the risk of the main outcomes, that is, $\mathrm{CAD}$ and stroke, among the three cohorts. The risk factors for AD and DLB were also evaluated. The baseline characteristics among the three groups were tested using the chi-square test and ANOVA. Logistic regression was used to evaluate the probable risk factors of $\mathrm{AD}$ and $\mathrm{DLB}$. We examined whether the risk of stroke and CAD increases among $A D$ and DLB patients using the Cox proportional hazard regression. A $p$ value less than 0.05 was significant. All the statistical analyses were conducted using the statistical software package, SAS, version 9.4 (SAS Institute Inc., Cary, NC).

\section{Results}

The final study population consisted of 7544 NC individuals, $1324 \mathrm{AD}$ patients, and $562 \mathrm{DLB}$ patients (Table 1). The number of female participants was higher than male participants in the AD cohort. Contrarily, the DLB cohort included a higher number of male participants. The difference in the average ages among the three cohorts was insignificant ( $p$ value $=0.26$ ). Participants under 65 years were less than $20 \%$ in each cohort. The distributions of baseline comorbidities differed among the $\mathrm{NC}, \mathrm{AD}$, and DLB groups ( $p$ value $<0.001$ ). The medication status was also significantly different $(p$ value $<0.01$ ) among the cohorts. The proportions of diabetes mellitus and hyperlipidemia were higher in AD patients than in DLB patients. There were $58.4 \%$ and $60.5 \%$ hypertensive individuals in the $\mathrm{AD}$ group and the DLB group, respectively.

Between AD and NC, compared to participants without any metabolic risk factors, the participants with one or more metabolic risk factors had significantly higher odds of $\mathrm{AD}$ 


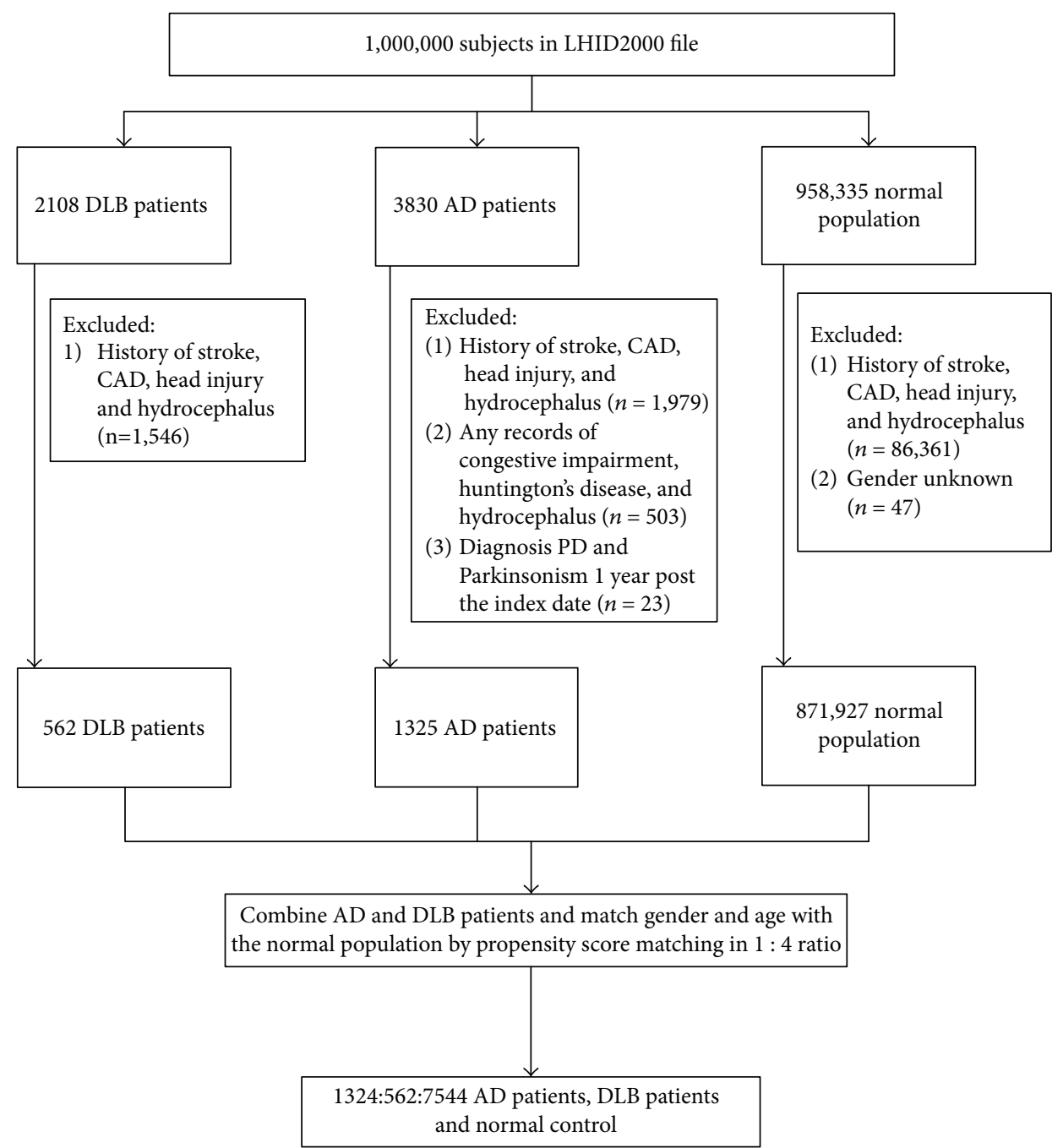

Figure 1: Enrollment flow chart for the study population.

TABLE 1: Demographic and background characteristics of NC, AD, and DLB patients.

\begin{tabular}{|c|c|c|c|c|}
\hline & $\mathrm{NC}$ & $\mathrm{AD}$ & DLB & $p$ value \\
\hline$N$ & 7544 & 1324 & 562 & \\
\hline Sex, f/m & $3758 / 3786$ & $768 / 556$ & $242 / 320$ & $<0.001$ \\
\hline \multicolumn{5}{|l|}{ Age } \\
\hline$<50$ & $216(2.9)$ & $39(2.9)$ & $15(2.7)$ & \\
\hline $50-64$ & $939(12.4)$ & $188(14.2)$ & $50(8.90)$ & \\
\hline$\geq 65$ & $6389(84.7)$ & $1097(82.9)$ & $497(88.4)$ & \\
\hline Mean (SD) & $74.5(11.1)$ & $74.2(11.4)$ & $75.1(10.3)$ & $0.26^{*}$ \\
\hline \multicolumn{5}{|l|}{ Comorbidity } \\
\hline Hypertension & $2777(36.8)$ & $773(58.4)$ & $340(60.5)$ & $<0.001$ \\
\hline Diabetes mellitus & $1132(15.0)$ & $387(29.2)$ & $142(25.3)$ & $<0.001$ \\
\hline Hyperlipidemia & $1201(15.9)$ & $466(35.2)$ & $146(26.0)$ & $<0.001$ \\
\hline \multicolumn{5}{|l|}{ Drug } \\
\hline Aspirin & $1139(15.1)$ & $468(35.3)$ & $186(33.1)$ & $<0.001$ \\
\hline Clopidogrel & $21(0.28)$ & $18(1.36)$ & $7(1.25)$ & $<0.001$ \\
\hline Warfarin & $45(0.6)$ & $20(1.51)$ & $5(0.89)$ & 0.002 \\
\hline
\end{tabular}

Chi-square test; *ANOVA. NC, normal control; AD, Alzheimer's disease; DLB, dementia with Lewy bodies; SD, standard deviation. 
TABLE 2: Risk factors for AD and DLB patients.

\begin{tabular}{|c|c|c|c|}
\hline \multirow{2}{*}{ Risk factors } & \multicolumn{3}{|c|}{ Multivariable ORs (95\% CI) } \\
\hline & $\mathrm{AD}$ versus $\mathrm{NC}$ & DLB versus NC & DLB versus $\mathrm{AD}$ \\
\hline \multicolumn{4}{|l|}{$\overline{\text { Sex }}$} \\
\hline Female & 1 (reference) & 1 (reference) & 1 (reference) \\
\hline Male & $0.82(0.72-0.92)^{* *}$ & $1.42(1.19-1.69)^{* * *}$ & $1.75(1.43-2.15)^{* * *}$ \\
\hline Age, per year & $1.0(0.99-1.0)$ & $1.0(0.99-1.01)$ & $1(0.99-1.01)$ \\
\hline \multicolumn{4}{|c|}{ Combination of metabolic risk factors (hypertension, diabetes, and hyperlipidemia) } \\
\hline$(---)$ & 1 (reference) & 1 (reference) & 1 (reference) \\
\hline$(+--)$ & $2.29(1.95-2.69)^{* * *}$ & $2.74(2.2-3.42)^{* * *}$ & $1.16(0.89-1.52)$ \\
\hline$(-+-)$ & $2.28(1.64-3.18)^{* * *}$ & $2.23(1.38-3.62)^{* * *}$ & $0.91(0.52-1.6)$ \\
\hline$(--+)$ & $3.7(2.78-4.93)^{* * *}$ & $2.66(1.65-4.29)^{* * *}$ & $0.66(0.39-1.11)$ \\
\hline$(++-)$ & $3.08(2.42-3.92)^{* * *}$ & $3.6(2.6-4.99)^{* * *}$ & $1.18(0.81-1.73)$ \\
\hline$(+-+)$ & $3.63(2.94-4.49)^{* * *}$ & $3.25(2.37-4.46)^{* * *}$ & $0.88(0.62-1.26)$ \\
\hline$(-++)$ & $3.58(2.48-5.17)^{* * *}$ & $2.14(1.1-4.17)^{*}$ & $0.62(0.3-1.28)$ \\
\hline$(+++)$ & $4.9(4.01-5.99)^{* * *}$ & $3.64(2.65-5)^{* * *}$ & $0.7(0.5-0.99)$ \\
\hline
\end{tabular}

+, presence; -, absence; CI, confidential interval; OR, odds ratio; NC, normal control; AD, Alzheimer's disease; DLB, dementia with Lewy bodies. Adjusted sex and age in logistic regression. ${ }^{*} p<0.05,{ }^{* *} p<0.01$, and ${ }^{* * *} p<0.001$.

(Table 2). The odds ratio of $\mathrm{AD}$ was 4.9 (95\% CI = 4.01-5.99, $p$ value $<0.001)$ in patients with three metabolic risk factors. For DLB and NC, the logistic regression showed an odds ratio of 3.64 for DLB $(95 \% \mathrm{CI}=2.65-5, p$ value $<0.001)$ in patients with three metabolic risk factors. Between DLB and $\mathrm{AD}$, there were no significant odds of DLB in patients with any metabolic risk factors. The male patients had low odds of $\mathrm{AD}(\mathrm{OR}=0.82,95 \% \mathrm{CI}=0.72-0.92$, $p$ value $<0.01)$, but high odds of DLB $(\mathrm{OR}=1.42,95 \% \mathrm{CI}=1.19-1.69$, $p$ value $<0.001)$.

The results of the Cox model indicated that the $\mathrm{AD}$ patients had a lower risk of outcomes ( $\mathrm{aHR}=0.67,95 \%$ $\mathrm{CI}=0.59-0.76$, $p$ value $<0.001)$ than the NC population (Table 3). Separated, the risks of CAD and stroke were 0.59 -fold $(95 \% \mathrm{CI}=0.51-0.69, p$ value $<0.001)$ and 1.02 fold $(95 \% \mathrm{CI}=0.8-1.3, p$ value $>0.05)$, respectively, in $\mathrm{AD}$ patients. Compared with the NC cohort, there was a higher stroke risk in the DLB group $(\mathrm{aHR}=2.08,95 \%$ $\mathrm{CI}=1.58-2.73$, $p$ value $<0.001)$. The risk of both outcomes combined was also higher $(\mathrm{aHR}=1.23,95 \% \mathrm{CI}=1.06-1.44$, $p$ value $<0.01)$. However, the risk of $\mathrm{CAD}$ alone was negligible $(\mathrm{aHR}=1.04,95 \% \quad \mathrm{CI}=0.86-1.24, \quad p \quad$ value $>0.05)$ among DLB patients. The risk of outcomes in males was higher than that in females $(\mathrm{aHR}=1.14,95 \% \mathrm{CI}=1.06-$ $1.23, p$ value $<0.001)$. The risk of outcomes increased 1.03 -fold per year with increasing age (95\% CI $=1.03-1.04$, $p$ value $<0.001)$. The aHR outcomes of hypertension and diabetes mellitus were $1.39(95 \% \mathrm{CI}=1.28-1.51$, $p$ value $<0.001)$ and $1.2(95 \% \mathrm{CI}=1.09-1.34, p$ value $<0.001)$, respectively. Hyperlipidemia reduced the risk of stroke $(\mathrm{aHR}=0.7,95 \%$ $\mathrm{CI}=0.55-0.9, p$ value $<0.01)$, but did not affect the risk of outcomes $(\mathrm{aHR}=0.9,95 \% \mathrm{CI}=0.81-1, p$ value $>0.05)$ and $\mathrm{CAD}(\mathrm{aHR}=0.96,95 \% \mathrm{CI}=0.86-1.09, p$ value $>0.05)$. There was no significant effect on the risk of outcomes with aspirin $(\mathrm{aHR}=0.98,95 \% \mathrm{CI}=0.89-1.09, p$ value $>0.05)$, clopidogrel $(\mathrm{aHR}=1.25, \quad 95 \% \mathrm{CI}=0.72-2.16, \quad p \quad$ value $>0.05)$, and warfarin $(\mathrm{aHR}=0.66,95 \% \mathrm{CI}=0.38-1.13, p$ value $>0.05)$. Additionally, we divided stroke outcome into ischemic and hemorrhagic stroke. The ischemic stroke risk was insignificant among $\mathrm{AD}$ patients $(\mathrm{aHR}=1.01,95 \% \mathrm{CI}=0.77-1.33$, $p$ value $>0.05$ ) when compared to the $\mathrm{NC}$ population (Table 4). In contrast, the DLB group had a higher ischemic stroke risk $\quad(\mathrm{aHR}=2.27, \quad 95 \% \quad \mathrm{CI}=1.68-3.06, \quad p$ value $<0.001)$. The aHRs of hemorrhagic stroke among AD and $\mathrm{DLB}$ patients were insignificant (for $\mathrm{AD}: \mathrm{aHR}=1.04$, $95 \% \mathrm{CI}=0.61-1.77, p$ value $>0.05$; for $\mathrm{DLB}: \mathrm{aHR}=1.4,95 \%$ $\mathrm{CI}=0.7-2.8, p$ value $>0.05)$.

\section{Discussion}

In this retrospective cohort study, female patients were more likely to have $\mathrm{AD}$, while male patients were more likely to have DLB. Patients with any one of the metabolic risk factors considered, had an increased risk of having AD or DLB. Patients who had three comorbid risk factors (hypertension, diabetes mellitus, and hyperlipidemia) simultaneously were at higher risk of having AD or DLB than patients with a single risk factor. The consequences of metabolic risk factors for $\mathrm{AD}$ are similar to those for DLB. Patients with AD had a lower risk, while patients with DLB had a higher risk of all CVD outcomes than the NC population. When outcomes were separated, patients with $\mathrm{AD}$ had a lower risk of CAD, and patients with DLB had a higher risk of ischemic stroke than the NC population. Patients with AD or DLB had no increased risk of hemorrhagic stroke.

We found that $\mathrm{AD}$ was more prevalent among female patients, while DLB was more prevalent among male patients. Sex differences between AD and DLB have been reported [13]. The age at onset of $\mathrm{AD}$ may be lower in women than in men with the same copies of the APOE $\varepsilon 4$ allele [14]. Male sex is a known risk factor for DLB $[3,5]$. 
TABLE 3: Multivariable analysis of risk of CAD and stroke in Cox proportional hazard regression.

\begin{tabular}{|c|c|c|c|}
\hline Risk factors & $\begin{array}{c}\text { All outcomes } \\
\text { Adjusted HR (95\% CI) }\end{array}$ & $\begin{array}{c}\text { CAD } \\
\text { Adjusted HR (95\% CI) }\end{array}$ & $\begin{array}{c}\text { Stroke } \\
\text { Adjusted HR }(95 \% \mathrm{CI})\end{array}$ \\
\hline $\mathrm{NC}$ & 1 (reference) & 1 (reference) & 1 (reference) \\
\hline $\mathrm{AD}$ & $0.67(0.59-0.76)^{* * *}$ & $0.59(0.51-0.69)^{* * *}$ & $1.02(0.8-1.3)$ \\
\hline DLB & $1.23(1.06-1.44)^{* *}$ & $1.04(0.86-1.24)$ & $2.08(1.58-2.73)^{* * *}$ \\
\hline \multicolumn{4}{|l|}{ Sex } \\
\hline Female & 1 (reference) & 1 (reference) & 1 (reference) \\
\hline Male & $1.14(1.06-1.23)^{* * *}$ & $1.11(1.02-1.21)^{*}$ & $1.26(1.07-1.49)^{* *}$ \\
\hline Age, per year & $1.03(1.03-1.04)^{* * *}$ & $1.03(1.02-1.03)^{* * *}$ & $1.05(1.04-1.06)^{* * *}$ \\
\hline Hypertension (yes versus no) & $1.39(1.28-1.51)^{* * *}$ & $1.39(1.27-1.53)^{* * *}$ & $1.37(1.14-1.64)^{* * *}$ \\
\hline Diabetes mellitus (yes versus no) & $1.2(1.09-1.34)^{* * *}$ & $1.15(1.02-1.29)^{*}$ & $1.43(1.15-1.78)^{* *}$ \\
\hline Hyperlipidemia (yes versus no) & $0.9(0.81-1)$ & $0.96(0.86-1.09)$ & $0.7(0.55-0.9)^{* *}$ \\
\hline Aspirin (yes versus no) & $0.98(0.89-1.09)$ & $0.95(0.85-1.07)$ & $1.1(0.89-1.37)$ \\
\hline Clopidogrel (yes versus no) & $1.25(0.72-2.16)$ & $1.29(0.69-2.41)$ & $1.14(0.36-3.58)$ \\
\hline Warfarin (yes versus no) & $0.66(0.38-1.13)$ & $0.6(0.31-1.15)$ & $0.84(0.31-2.27)$ \\
\hline
\end{tabular}

HR, hazard ratio; CI, confidence interval; NC, normal control; AD, Alzheimer's disease; DLB, dementia with Lewy bodies. Adjusted sex, age, hypertension, diabetes mellitus, hyperlipidemia, aspirin, clopidogrel, and warfarin in Cox proportional hazards regression. ${ }^{*} p<0.05,{ }^{* *} p<0.01$, and ${ }^{* * *} p<0.001$.

TABLE 4: Multivariable analysis of the risk of ischemic stroke and hemorrhagic stroke in Cox proportional hazard regression.

\begin{tabular}{lcc}
\hline Risk factors & $\begin{array}{c}\text { Ischemic stroke } \\
\text { Adjusted HR (95\% CI) }\end{array}$ & $\begin{array}{c}\text { Hemorrhage stroke } \\
\text { Adjusted HR (95\% CI) }\end{array}$ \\
\hline NC & 1 (reference) & 1 (reference) \\
AD & $1.01(0.77-1.33)$ & $1.04(0.61-1.77)$ \\
DLB & $2.27(1.68-3.06)^{* * *}$ & $1.4(0.7-2.8)$ \\
Sex & & 1 (reference) \\
$\quad$ Female & 1 (reference) & $1.74(1.21-2.49)^{* *}$ \\
$\quad$ Male & $1.16(0.97-1.4)$ & $1.05(1.03-1.07)^{* * *}$ \\
Age, per year & $1.05(1.04-1.06)^{* * *}$ & $1.5(1.03-2.19)^{*}$ \\
Hypertension (yes versus no) & $1.34(1.09-1.64)^{* *}$ & $1.25(0.76-2.06)$ \\
Diabetes mellitus (yes versus no) & $1.48(1.16-1.88)^{* *}$ & $0.52(0.29-0.95)^{*}$ \\
Hyperlipidemia (yes versus no) & $0.75(0.57-0.98)^{*}$ & $0.76(0.46-1.27)$ \\
Aspirin (yes versus no) & $1.21(0.95-1.53)$ & $2.36(0.32-17.36)$ \\
Clopidogrel (yes versus no) & $0.91(0.22-3.68)$ & \\
Warfarin (yes versus no) & $1.05(0.39-2.82)$ & \\
\hline
\end{tabular}

HR, hazard ratio; CI, confidence interval; NC, normal control; AD, Alzheimer's disease; DLB, dementia with Lewy bodies. Adjusted sex, age, hypertension, diabetes mellitus, hyperlipidemia, aspirin, clopidogrel, and warfarin in Cox proportional hazards regression. ${ }^{*} p<0.05,{ }^{* *} p<0.01$, and ${ }^{* * *} p<0.001$.

The cause of these sex differences remains uncertain and need to be clarified.

We found that hypertension significantly increased the risk of both $\mathrm{AD}(\mathrm{OR}=2.29,95 \% \mathrm{CI}=1.95-2.69)$ and $\mathrm{DLB}$ $(\mathrm{OR}=2.74,95 \% \mathrm{CI}=2.20-3.42)$. Hypertension is a risk factor for dementia [9]. Studies have shown that hypertension increases the risk of $\mathrm{AD}$, and that antihypertensive treatment can reduce the risk of $\mathrm{AD}$ [10]. The relationship between hypertension and DLB is less understood. In a neuropathological study, patients with DLB pathology were found to have a significantly higher incidence of hypertension [15].
We also found that diabetes mellitus increased the risk of $\mathrm{AD}(\mathrm{OR}=2.28,95 \% \mathrm{CI}=1.64-3.18)$ and $\mathrm{DLB}(\mathrm{OR}=2.23$, $95 \% \mathrm{CI}=1.38-3.62)$. Diabetes mellitus is a risk factor for cognitive decline [10]. Insulin insufficiency increases the risk of $\mathrm{AD}$ [10]. Either an excessive glucose level or an insulin dysfunction may play a role in $\mathrm{AD}$ [10]. There is limited information about the effect of diabetes on DLB. Although diabetes was a risk factor for DLB in our study, it did not alter the risk of DLB in another study [5]. This conflicting result may have arisen because of the different diagnostic criteria for diabetes in different populations, and further studies are warranted to clarify if diabetes causes DLB. 
Hyperlipidemia was another risk factor that increased $\mathrm{AD}(\mathrm{OR}=3.70,95 \% \mathrm{CI}=2.78-4.93)$ and $\mathrm{DLB}(\mathrm{OR}=2.66$, $95 \% \mathrm{CI}=1.65-4.29$ ). Dysfunction of lipid metabolism plays an important role in dementia. High total serum cholesterol levels are associated with dementia and AD [10]. APOE is an important class of lipoprotein in lipid metabolism, and APOE4, an allele of APOE, is implicated in AD. APOE4 is also more frequent in DLB patients than in controls [5]. Nevertheless, the mechanism by which lipid disorders affect DLB is obscure. Considering its close association with dementia and that it is a modifiable risk factor, we suggest that studies focus more on lipid disorders in future DLB studies.

Having three metabolic risk factors simultaneously further increased the risk of $\mathrm{AD}(\mathrm{OR}=4.90,95 \% \mathrm{CI}=4.01-$ 5.99) and DLB $(\mathrm{OR}=3.64,95 \% \mathrm{CI}=2.65-5.00)$. The hazard of metabolic risk factors is similar in AD and DLB. AD and DLB share many similar risk factors such as depression, smoking, and the APOE $\varepsilon 4$ allele [5]. Cerebrovascular lesions are also frequent in both AD and DLB $[16,17]$. AD and DLB show clinical and pathological similarity, and metabolic risk factors may have similar roles in the risk of developing $\mathrm{AD}$ and DLB. Summarizing, better lifestyle modifications and medication for the treatment of metabolic risk factors may not only prevent stroke and CAD but also AD and DLB.

In a risk-adjusted long-term follow-up, we found that $\mathrm{AD}$ had a decreasing risk of CAD $(\mathrm{aHR}=0.59,95 \% \mathrm{CI}=0.51-$ 0.69 ), while DLB had an increasing risk of ischemic stroke $(\mathrm{aHR}=2.27,95 \% \mathrm{CI}=1.68-3.06) . \mathrm{AD}$ is associated with atherosclerosis [18]. Evidence has shown that cerebral atherosclerosis, involving extracranial or intracranial arteries, contributes to the cognitive decline in $\mathrm{AD}[19,20]$. Coronary atherosclerosis also worsens cognitive function in $\mathrm{AD}$ [11]. These facts reflect the hemodynamic compromise or hypoperfusion of the brain which correlates with $\mathrm{AD}$ deterioration [21-23]. However, AD itself does not necessarily increase the risk of CAD. In fact, a recent study showed that APOE $\varepsilon 4$ does not interact with CAD [24]. Interestingly, our results implied that patients with $\mathrm{AD}$ might have protective factors to $\mathrm{CAD}$, with the underdiagnosis of $\mathrm{CAD}$ or ischemic stroke in $\mathrm{AD}$ having been one possible explanation. However, we did not find a similar effect in DLB, making it less likely. A change in eating behaviour in $\mathrm{AD}$ was another possible explanation. Anorexia and weight loss, which are common in advanced $\mathrm{AD}$ [25], may reduce blood sugar or lipid level and decrease the risk of atherosclerosis. A change in amyloid $\beta(\mathrm{A} \beta)$ metabolism may affect the risk of CAD in AD. Extracellular deposition of $A \beta$ in the brain is the pathological hallmark of $\mathrm{AD}$ [26]. A dysfunction of $\mathrm{A} \beta$ production and clearance in the central nervous system may contribute to the accumulation of cerebral $\mathrm{A} \beta$ in late onset $\mathrm{AD}[27,28]$. Higher plasma $\mathrm{A} \beta$ also correlates with cerebral white matter lesions and ischemic heart disease [29]. In short, the change in the $\mathrm{A} \beta$ metabolic pathway in $\mathrm{AD}$ may be associated with cerebrovascular and cardiovascular risks, and further investigations are warranted.

A history of stroke is more frequently observed in patients with DLB [5]. White matter hyperintensities on cerebral T2-weighted magnetic resonance imaging are higher in DLB than in $\mathrm{AD}$ patients [17]. DLB usually shows hypoperfusion in the striatum and the visual cortex [3]. All these studies suggest that DLB correlates with the function of cerebral small vessels. Postural hypotension is commonly seen in DLB [3], and is also a potential risk factor for ischemic stroke. Intracellular $\alpha$-synuclein aggregation is characteristic of DLB [1]. Studies also show a potential role for $\alpha$-synuclein in ischemic stroke modulation [30]. Further studies are required to determine the mechanism of stroke in DLB.

Cerebral microbleeds are small hemosiderin deposits in the brain and are not unusual in AD or DLB [31, 32]. The gradual spreading of the intracellular aggregation of hyperphosphorylated tau (P-tau) in the brain is associated with $\mathrm{AD}$ progression [1]. Studies also reveal the association between cerebral microbleeds and P-tau [33, 34]. Interestingly, our data did not reveal increased hemorrhagic stroke in both $\mathrm{AD}$ and $\mathrm{DLB}$ patients when compared to NC individuals. This may imply that the pathological processes for intracerebral hemorrhage and cerebral microbleeds may differ in AD and DLB.

Limitations were inevitable in this study. First, subjects were classified based on the main clinical diagnosis and bias from misclassification was possible. Dementia is a complex syndrome and accurate diagnosis is challenging because of the overlapping clinical presentation. Although we used multiple methods during the inclusion process to identify the diagnosis and to minimize misclassification, a few atypical cases may still present difficulties in classification. Second, the severity of metabolic risk factors and their different treatment methods can potentially affect the risk of CAD or stroke. Third, this was a Taiwan-based study, and the influence of risk may vary among different racial groups. Fourth, this was a retrospective study, and selection and information biases cannot be completely excluded.

\section{Conclusions}

Hypertension, diabetes mellitus, and hyperlipidemia are risk factors of AD and DLB. Multiple metabolic risk factors, when simultaneously present, might aggravate the risk of $\mathrm{AD}$ and DLB. The control of metabolic risk factors is crucial for the prevention of $\mathrm{AD}$ and $\mathrm{DLB}$. Patients with $\mathrm{AD}$ might have a lower risk of CAD, while patients with DLB might have a higher risk of ischemic stroke. Our study suggests that better lifestyle modifications and medications for the treatment of metabolic risk factors may lower the risk of $\mathrm{AD}$ and DLB. Furthermore, stroke prevention is important in patients with DLB. Further studies to understand the mechanism of stroke in DLB are warranted.

\section{Data Availability}

The data used to support the findings of this study are available from the corresponding author upon request.

\section{Conflicts of Interest}

The authors declare that they have no conflicts of interest. 


\section{Acknowledgments}

This work was supported by grants from the Ministry of Health and Welfare, Taiwan (MOHW107-TDU-B-212123004), China Medical University Hospital, Academia Sinica Stroke Biosignature Project (BM10701010021), MOST Clinical Trial Consortium for Stroke (MOST 106-2321-B039-005), Tseng-Lien Lin Foundation, Taichung, Taiwan, and Katsuzo and Kiyo Aoshima Memorial Funds, Japan.

\section{References}

[1] F. M. Elahi and B. L. Miller, "A clinicopathological approach to the diagnosis of dementia," Nature Reviews Neurology, vol. 13, no. 8, pp. 457-476, 2017.

[2] B. V. Zlokovic, "Neurovascular pathways to neurodegeneration in Alzheimer's disease and other disorders," Nature Reviews Neuroscience, vol. 12, no. 12, pp. 723-738, 2011.

[3] Z. Walker, K. L. Possin, B. F. Boeve, and D. Aarsland, "Lewy body dementias," The Lancet, vol. 386, no. 10004, pp. 16831697, 2015.

[4] I. G. McKeith, B. F. Boeve, D. W. Dickson et al., "Diagnosis and management of dementia with Lewy bodies: fourth consensus report of the DLB Consortium," Neurology, vol. 89, no. 1, pp. 88-100, 2017.

[5] B. P. Boot, C. F. Orr, J. E. Ahlskog et al., "Risk factors for dementia with Lewy bodies: a case-control study," Neurology, vol. 81, no. 9, pp. 833-840, 2013.

[6] R. E. Mrak and W. S. Griffin, "Dementia with Lewy bodies: definition, diagnosis, and pathogenic relationship to Alzheimer's disease," Neuropsychiatric disease and treatment, vol. 3, no. 5, pp. 619-625, 2007.

[7] T. B. Chen, S. Y. Yiao, Y. Sun et al., "Comorbidity and dementia: a nationwide survey in Taiwan," PLoS One, vol. 12, no. 4, article e0175475, 2017.

[8] S. Kaffashian, A. Dugravot, A. Elbaz et al., "Predicting cognitive decline: a dementia risk score vs the Framingham vascular risk scores," Neurology, vol. 80, no. 14, pp. 1300-1306, 2013.

[9] F. W. Unverzagt, L. A. McClure, V. G. Wadley et al., "Vascular risk factors and cognitive impairment in a stroke-free cohort," Neurology, vol. 77, no. 19, pp. 1729-1736, 2011.

[10] C. Y. Santos, P. J. Snyder, W. C. Wu, M. Zhang, A. Echeverria, and J. Alber, "Pathophysiologic relationship between Alzheimer's disease, cerebrovascular disease, and cardiovascular risk: a review and synthesis," Alzheimer's \& Dementia: Diagnosis, Assessment \& Disease Monitoring, vol. 7, pp. 69-87, 2017.

[11] M. Bleckwenn, L. Kleineidam, M. Wagner et al., "Impact of coronary heart disease on cognitive decline in Alzheimer's disease: a prospective longitudinal cohort study in primary care," British Journal of General Practice, vol. 67, no. 655, pp. e111-e117, 2017.

[12] J. Attems and K. A. Jellinger, "The overlap between vascular disease and Alzheimer's disease-lessons from pathology," BMC Medicine, vol. 12, no. 1, p. 206, 2014.

[13] S. A. Vann Jones and J. T. O’Brien, "The prevalence and incidence of dementia with Lewy bodies: a systematic review of population and clinical studies," Psychological Medicine, vol. 44, no. 4, pp. 673-683, 2014.

[14] S. C. Neu, J. Pa, W. Kukull et al., "Apolipoprotein E genotype and sex risk factors for Alzheimer disease: a meta-analysis," JAMA Neurology, vol. 74, no. 10, pp. 1178-1189, 2017.
[15] J. De Reuck, V. Deramecourt, C. Cordonnier, D. Leys, F. Pasquier, and C. A. Maurage, "Prevalence of small cerebral bleeds in patients with a neurodegenerative dementia: a neuropathological study," Journal of the Neurological Sciences, vol. 300, no. 1-2, pp. 63-66, 2011.

[16] K. A. Jellinger, "Prevalence and impact of cerebrovascular lesions in Alzheimer and Lewy body diseases," Neurodegenerative Diseases, vol. 7, no. 1-3, pp. 112-115, 2010.

[17] L. Sarro, N. Tosakulwong, C. G. Schwarz et al., "An investigation of cerebrovascular lesions in dementia with Lewy bodies compared to Alzheimer's disease," Alzheimer's \& Dementia, vol. 13, no. 3, pp. 257-266, 2017.

[18] A. Hofman, A. Ott, M. M. B. Breteler et al., "Atherosclerosis, apolipoprotein E, and prevalence of dementia and Alzheimer's disease in the Rotterdam Study," The Lancet, vol. 349, no. 9046, pp. 151-154, 1997.

[19] A. E. Roher, S. L. Tyas, C. L. Maarouf et al., "Intracranial atherosclerosis as a contributing factor to Alzheimer's disease dementia," Alzheimer's \& Dementia, vol. 7, no. 4, pp. 436444, 2011.

[20] M. Silvestrini, B. Gobbi, P. Pasqualetti et al., "Carotid atherosclerosis and cognitive decline in patients with Alzheimer's disease," Neurobiology of Aging, vol. 30, no. 8, pp. 11771183, 2009.

[21] A. E. Roher, Z. Garami, S. L. Tyas et al., “Transcranial Doppler ultrasound blood flow velocity and pulsatility index as systemic indicators for Alzheimer's disease," Alzheimer's \& Dementia, vol. 7, no. 4, pp. 445-455, 2011.

[22] A. Salminen, A. Kauppinen, and K. Kaarniranta, "Hypoxia/ ischemia activate processing of amyloid precursor protein: impact of vascular dysfunction in the pathogenesis of Alzheimer's disease," Journal of Neurochemistry, vol. 140, no. 4, pp. 536-549, 2017.

[23] L. Qiu, G. Ng, E. K. Tan, P. Liao, N. Kandiah, and L. Zeng, "Chronic cerebral hypoperfusion enhances Tau hyperphosphorylation and reduces autophagy in Alzheimer's disease mice," Scientific Reports, vol. 6, no. 1, article 23964, 2016.

[24] D. M. Lyall, J. Ward, S. J. Ritchie et al., "Alzheimer disease genetic risk factor $A P O E$ e4 and cognitive abilities in 111,739 UK Biobank participants," Age and Ageing, vol. 45, no. 4, pp. 511-517, 2016.

[25] S. Gillette-Guyonnet, F. Nourhashémi, S. Andrieu et al., "Weight loss in Alzheimer disease," The American Journal of Clinical Nutrition, vol. 71, no. 2, pp. 637S-642S, 2000.

[26] F. L. Heppner, R. M. Ransohoff, and B. Becher, "Immune attack: the role of inflammation in Alzheimer disease," Nature Reviews Neuroscience, vol. 16, no. 6, pp. 358-372, 2015.

[27] A. Ramanathan, A. R. Nelson, A. P. Sagare, and B. V. Zlokovic, "Impaired vascular-mediated clearance of brain amyloid beta in Alzheimer's disease: the role, regulation and restoration of LRP1," Frontiers in Aging Neuroscience, vol. 7, p. 136, 2015.

[28] H. J. Ahn, D. Zamolodchikov, M. Cortes-Canteli, E. H. Norris, J. F. Glickman, and S. Strickland, “Alzheimer's disease peptide $\beta$-amyloid interacts with fibrinogen and induces its oligomerization," Proceedings of the National Academy of Sciences of the United States of America, vol. 107, no. 50, pp. 21812-21817, 2010.

[29] S. Janelidze, E. Stomrud, S. Palmqvist et al., "Plasma $\beta$-amyloid in Alzheimer's disease and vascular disease," Scientific Reports, vol. 6, no. 1, article 26801, 2016. 
[30] T. H. Kim and R. Vemuganti, "Mechanisms of Parkinson's disease-related proteins in mediating secondary brain damage after cerebral ischemia," Journal of Cerebral Blood Flow \& Metabolism, vol. 37, no. 6, pp. 1910-1926, 2017.

[31] A. A. Sepehry, D. Lang, G. Y. Hsiung, and A. Rauscher, "Prevalence of brain microbleeds in Alzheimer disease: a systematic review and meta-analysis on the influence of neuroimaging techniques," American Journal of Neuroradiology, vol. 37, no. 2, pp. 215-222, 2016.

[32] S. W. Kim, S. J. Chung, Y.-S. Oh et al., "Cerebral microbleeds in patients with dementia with Lewy bodies and Parkinson disease dementia," American Journal of Neuroradiology, vol. 36, no. 9, pp. 1642-1647, 2015.

[33] T. Poliakova, O. Levin, A. Arablinskiy, E. Vasenina, and I. Zerr, "Cerebral microbleeds in early Alzheimer's disease," Journal of Neurology, vol. 263, no. 10, pp. 1961-1968, 2016.

[34] G. C. Chiang, J. C. Cruz Hernandez, K. Kantarci, C. R. Jack, M. W. Weiner, and for the Alzheimer's Disease Neuroimaging Initiative, "Cerebral microbleeds, CSF p-Tau, and cognitive decline: significance of anatomic distribution," American Journal of Neuroradiology, vol. 36, no. 9, pp. 1635-1641, 2015. 


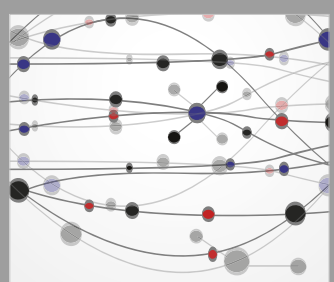

The Scientific World Journal
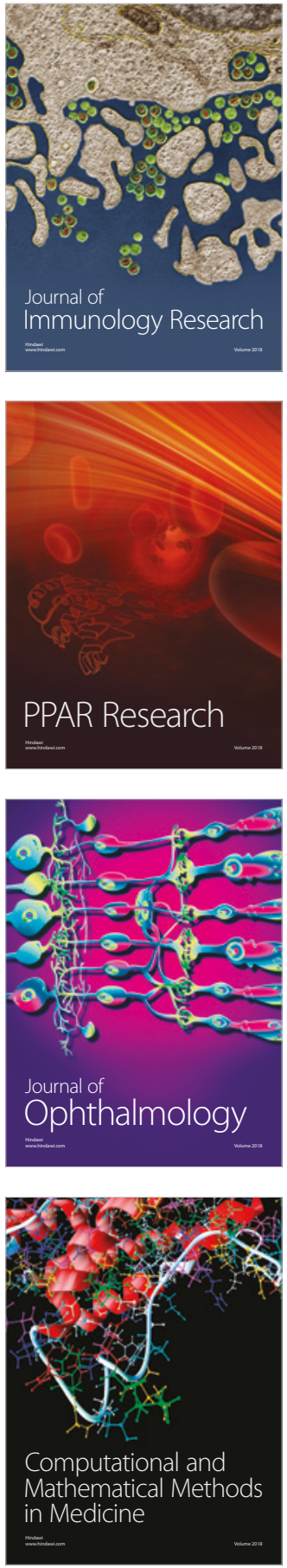

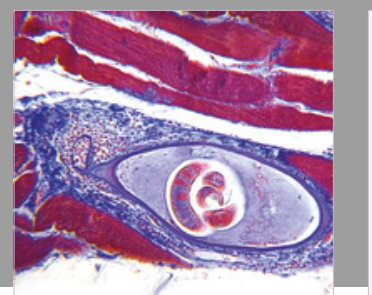

Gastroenterology Research and Practice

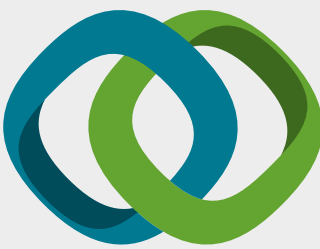

\section{Hindawi}

Submit your manuscripts at

www.hindawi.com
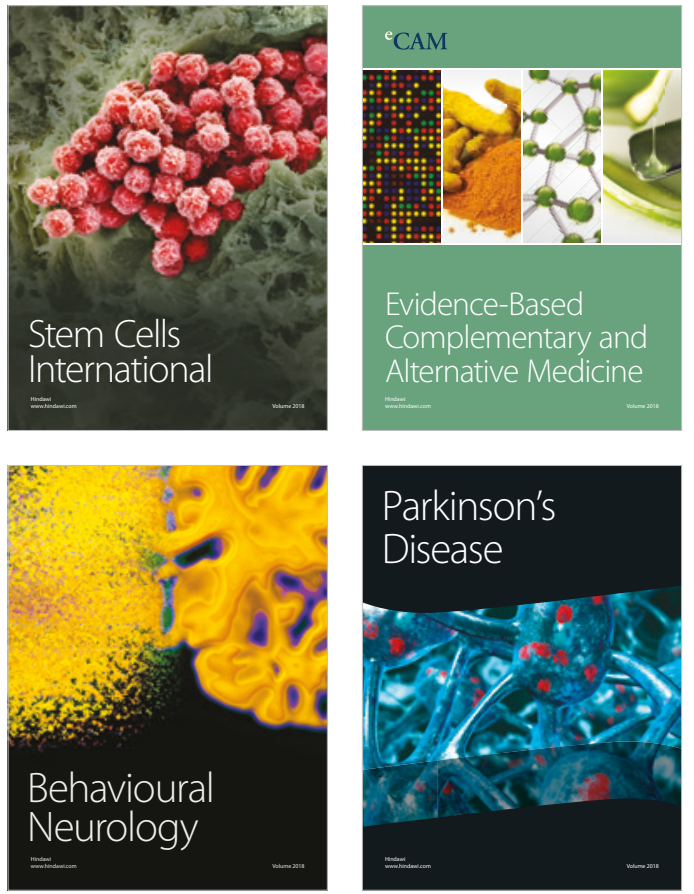

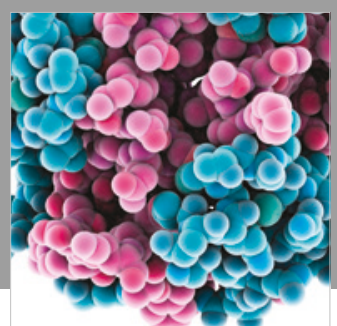

ournal of

Diabetes Research

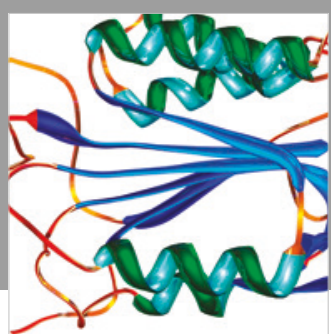

Disease Markers
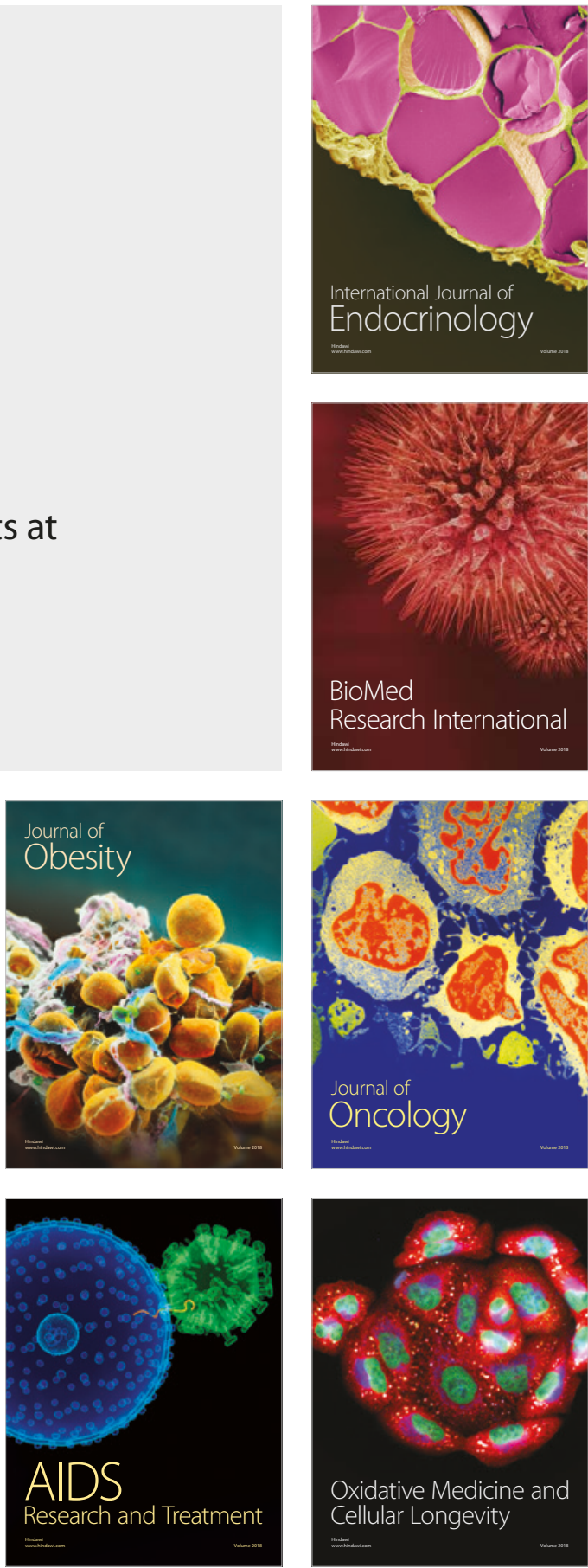